Rev Esp Casos Clin Med Intern (RECCMI). 2020 (Dic); 5(3): 109-111

\title{
Lupus eritematoso sistémico tardío asociado a síndrome de Sjögren
}

Sara Carrascosa-García', Miguel Ángel Moruno-Benita², Álvaro García-Campos'
'Servicio de Medicina Interna. Consorcio Hospital General Universitario Valencia. Valencia. España
'Servicio de Cardiología. Consorcio Hospital General Universitario Valencia. Valencia. España

Recibido: 16/06/2019

Aceptado: 14/10/2020

En línea: 31/12/2020

Citar como: Carrascosa-García S, Moruno-Benita MÁ, García-Campos Á. Lupus eritematoso sistémico tardío asociado a síndrome de Sjögren. Rev Esp Casos Clin Med Intern (RECCMI). 2020 (Dic); 5(3): 109-111. doi: 10.32818/reccmi.a5n3a3.

Cite this as: Carrascosa-García S, Moruno-Benita MÁ, García-Campos Á. Late Systemic Lupus Erythematosus associated with Sjögren's syndrome. Rev Esp Casos Clin Med Intern (RECCMI). 2020 (Dec); 5(3): 109-111. doi: 10.32818/reccmi.a5n3a3.

Autor para correspondencia: Miguel Ángel Moruno-Benita. mamoruno93@gmail.com

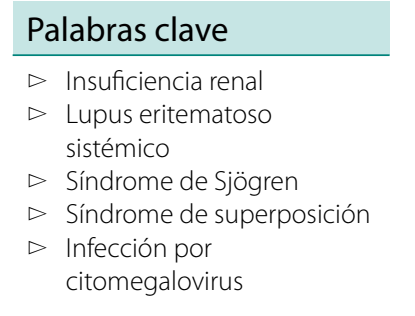

Keywords

$\triangleright$ Renal failure

$\triangleright$ Systemic Lupus Erythematosus

$\triangleright$ Sjögren Syndrome

$\triangleright$ Overlap Syndrome

$\triangleright$ Cytomegalovirus infection

\begin{abstract}
Resumen
Presentamos el caso de una paciente de edad avanzada, con diagnóstico previo de síndrome de Sjögren, con ingreso por deterioro de función renal y clínica de hematemesis. Durante el ingreso, presenta diarrea, de posible causa infecciosa, con detección de viremia de citomegalovirus y posterior mejoría tras antibioterapia y antivírico. El estudio etiológico evidencia el deterioro de la función renal de causa prerrenal por anemia y deshidratación; posrenal, por cambios morfológicos; y renal, por sedimento urinario patológico sospechoso de nefritis lúpica. Se realiza diagnóstico de sospecha de Lupus Eritematoso Sistémico de inicio tardío y de síndrome de superposición con síndrome de Sjögren. También se sospecha neoplasia gástrica subyacente.

Abstract
We report the case of an elderly woman, with a previous diagnosis of Sjögren's Syndrome with renal failure and haema-
temesis. During admission, she presented infectious complications (diarrhea), with detection of cytomegalovirus viremia
and subsequent improvement after antibiotic and antiviral therapy. The final diagnosis was an acute kidney injury due
to a prerenal component because of low water intake and anaemia, postrenal component as a result of morphological
changes, and intrinsic renal component on account of suspected lupus nephropathy (because of pathological urinary
sediment). The suspected diagnosis was late-onset Systemic Lupus Erythematosus and Overlap Syndrome. Furthermore,
underlying gastric neoplasia was suspected.
\end{abstract}

Puntos destacados

$\triangleright$ Presentamos el caso de una paciente con deterioro de función renal multifactorial.

$\triangleright$ La relevancia del caso reside en la necesidad de un estudio etiológico completo en pacientes de edad avanzada, sin descartar la presencia de una enfermedad autoinmune.

\section{Introducción}

Los síndromes de solapamiento o superposición (en inglés, overlap) son aquellos en los que coexisten manifestaciones clínicas o analíticas de dos o más enfermedades autoinmunes. Se deben sospechar ante cuadros clínicos abigarrados, sin criterios diagnósticos completos, sobre todo, en mujeres con diagnóstico previo de enfermedad autoinmune.

Exponemos el caso de una paciente anciana que, en el trascurso de un cuadro constitucional, posiblemente secundario a enfermedad neoplásica y varios episodios infecciosos, presenta deterioro de función renal y derrame pleural. Tras realizar un primer estudio para determinar el origen (renal, posrenal o prerrenal), y ante alteraciones analíticas compatibles, se sospecha un lupus eritematoso sistémico (LES) de inicio tardío y superposición con síndrome de Sjögren (SS).

\section{Caso clínico}

\section{Antecedentes}

Mujer de 81 años que refiere vómitos de dos días de evolución, con restos hemáticos frescos, sin disfagia, odinofagia ni dolor abdominal. Refiere anorexia, pérdida de peso subjetiva y astenia de dos meses de evolución, acompañados de xerostomía y xeroftalmia. Niega fiebre ni clínica infecciosa por aparatos. Niega alergias ni hábitos tóxicos. 
Carrascosa-García S, Moruno-Benita MÁ, García-Campos Á.

Entre sus antecedentes, destaca una fibrilación auricular, hipertensión arterial dislipemia y SS, en tratamiento con valsartan, bisoprolol, flecainida y 6 mg de metilprednisolona diarios.

Presenta úlceras en miembros inferiores de larga evolución, con varios ingresos por celulitis por Staphylococcus aureus, tratada con betalactámicos y quinolonas.

Es parcialmente dependiente para actividades básicas y no tiene deterioro cognitivo

\section{Exploración física}

Está caquéctica, deshidratada y pálida. La auscultación cardíaca, respiratoria y exploración abdominal son anodinas. Presenta varias úlceras a nivel pretibial derecho, no sobreinfectadas.

\section{Pruebas complementarias}

Se realiza batería de autoinmunidad con anticuerpos anticitoplasma neutrófilo negativo; ANA positivo 1/320; anticuerpos dsDNA 100,8 Ul/mL (positivo > 35 $\mathrm{UI} / \mathrm{mL})$; patrón IFI nuclear granular fino.

Anticuerpos antiRo52 y antiRo60 positivos; anticuerpos RNP positivo. Los anticuerpos SM, SS-b, Scl-70, Jo-1, anti-membrana basal glomerular y los anticentrómero son negativos.

Hay consumo de complemento, C3 41 mg/dL (85,0-193,0) y C4 2 mg/dL (12,0$36,0)$ y aumento de la velocidad de sedimentación globular, 61 mm/h $(1,0-20,0)$

Se realiza estudio de anemia, con test de Coombs Directo negativo, haptoglobina 297 mg/dL (30,0-200,0) e hiperferritinemia (2320 Mg/L, 10,0-120,0).

Se realiza estudio de orina de 24 horas con creatinuria 390 mg/24 h (10001800); proteinuria $0,4 \mathrm{~g} / 24 \mathrm{~h}(0,05-0,2)$ y albúmina $3,25 \mathrm{mg} / 24$ h $(0-30)$. Los cultivos de orina, sangre y heces fueron negativos.
El estudio de citomegalovirus (CMV) en sangre (Polymerase chain reaction, PCR) fue positivo: $1436 \mathrm{IU} / \mathrm{mL}$ (Tabla 1).

PEn la radiografía de tórax se evidencia derrame pleural bilateral (Figura 1), con ecocardiografía transtorácica sin alteraciones.

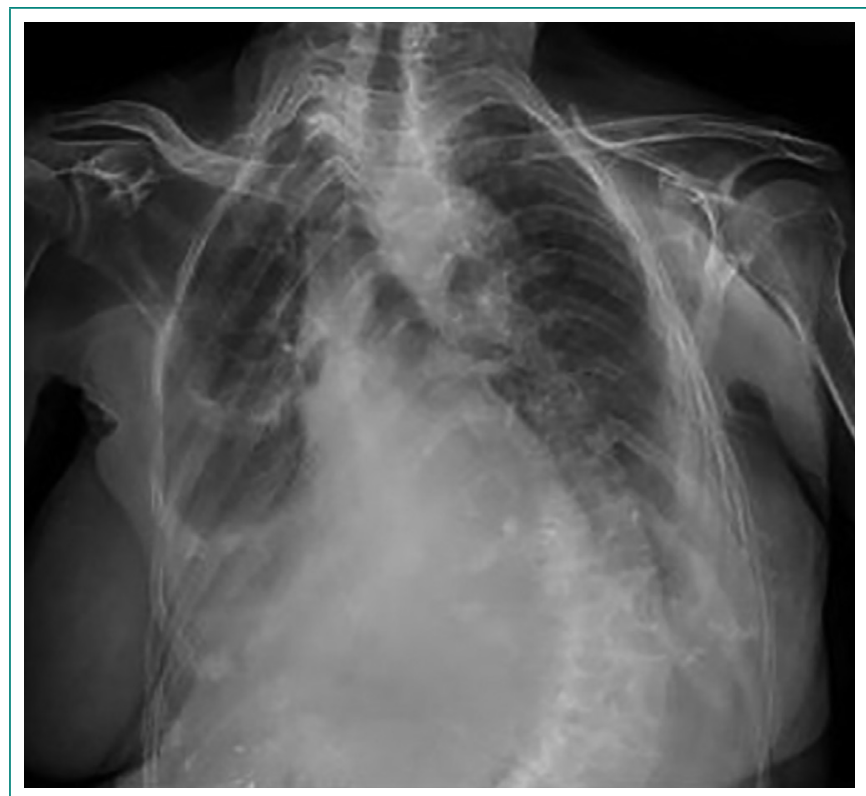

Figura 1. Radiografía de tórax.

En ecografía renal presenta dilatación de la pelvis renal y uréter proximal izquierdo, severa y de aspecto crónico.

En el estudio abdominal, se observa un engrosamiento de la pared gástrica de aspecto tumoral con discreta cantidad de líquido libre, datos confirmados mediante tomografía axial computerizada (TAC) sin contraste, que no aporta nueva información.

\begin{tabular}{|c|c|c|c|c|c|c|c|}
\hline & -30 días & -15 días & Ingreso & +5 días & +15 días & +30 días & Valores de referencia \\
\hline Hemoglobina (g/dL) & 11,6 & 8,3 & 9,7 & 11 & 9,2 & 9,4 & $12,5-16,0$ \\
\hline Hematocrito (\%) & 34,9 & 25,7 & 28,8 & 33,3 & 28,2 & 28,4 & $37,0-48,0$ \\
\hline Leucocitos $(10 * 9 / \mathrm{L})$ & 11 & 8,6 & 22,4 & 19,4 & 7,7 & 23 & $3,8-10,8$ \\
\hline Neutrófilos (10*9/L) & 8,9 & & 18,8 & 17,2 & 5,1 & 19,3 & $1,8-7,5$ \\
\hline Plaquetas (10*9/L) & 222 & 42 & 41 & 70 & 105 & 188 & $135,0-350,0$ \\
\hline Sodio (mEq/L) & 135 & 136 & 139 & 139 & 137 & 133 & $136-146$ \\
\hline Potasio (mEq/L) & 4,1 & 4,4 & 4,1 & 3,5 & 4,8 & 4,1 & $3,5-5,1$ \\
\hline Magnesio (mEq/L) & & & & 1,6 & 1,8 & 2,2 & $1,9-2,5$ \\
\hline Fósforo (mEq/L) & & 4,5 & & 4,3 & 6 & 6,1 & $2,5-4,5$ \\
\hline Calcio (mEq/L) & & 7,3 & 7 & 6,7 & 8,5 & 9,7 & $8,8-10,6$ \\
\hline Proteínas (g/dL) & & 6 & 5,2 & 5 & 4,5 & 4,2 & $6,6-8,3$ \\
\hline Creatinina (mg/dL) & 1,02 & 5,44 & 5,52 & 4,78 & 4,75 & 3,67 & $0,51-0,95$ \\
\hline Urea (mg/dL) & 62 & 226,3 & 251,5 & 182,1 & 215,4 & 207,5 & $17,0-43,0$ \\
\hline $\mathrm{FG}\left(\mathrm{mL} / \mathrm{min} / 1,73 \mathrm{~m}^{2}\right)$ & & 6,88 & 6,76 & 8,05 & 8,11 & 11 & \\
\hline Vitamina D - (25) OH D3 (ng/mL) & & & & 26,1 & & & $30-100$ \\
\hline Paratohormona (pg/mL) & & & & 404 & & & $12-88$ \\
\hline Proteína C Reactiva (PCR) (mg/dL) & 18,2 & & 10,5 & 17,6 & 1,4 & 4,2 & $0,0-0,5$ \\
\hline Procalcitonina (PCT) $(\mathrm{ng} / \mathrm{mL})$ & & & & 2,44 & 0,75 & & $0,0-0,05$ \\
\hline
\end{tabular}

Tabla 1. Evolución analítica. 


\section{Evolución}

Durante su estancia hospitalaria no presenta nuevos sangrados y se desestiman pruebas invasivas endoscópicas. Además, se constata fiebre diaria con deposiciones líquidas, sin aislamiento microbiológico en heces, iniciando antibioterapia con ceftriaxona y metronidazol intravenoso (iv) y posteriormente, meropenem (iv), hasta conseguir mejoría clínica y analítica.

Como es una paciente inmunodeprimida crónica, se solicita viremia de CMV siendo positiva, por lo que se añade ganciclovir (iv), con posterior negativización de la viremia, mejoría de la diarrea, sin observar lesiones en fondo de ojo.

Respecto a la ureterohidronefrosis, no se evidencia una causa justificable en las pruebas de imagen, desestimando la realización de otras medidas invasivas.

Analíticamente, destaca hipocomplementemia, ANA positivo, anti-DNAds elevado, antiRo y anti RNP positivo, pancitopenia, proteinuria y microhematuria que hace sospechar un lupus eritematoso sistémico, en paciente con diagnóstico previo de SS.

Tras estabilidad clínica y analítica, bajo tratamiento antibiótico y antiviral, y ante presencia de derrame pleural bilateral y empeoramiento de la disnea, se inicia metilprednisolona 500 mg (iv) durante tres días, con posterior pauta de 0,5 $\mathrm{mg} / \mathrm{kg} / \mathrm{d}$ ía, tras lo que aumenta el recuento plaquetario, permitiendo añadir hidroxicloroquina.

Con la mejoría clínica y sin infección activa, junto con estudio de serologías (hepatitis víricas y Virus de la Inmunodeficiencia Humana) y ensayo liberación de interferón gamma (IGRA) negativas, se inicia tratamiento con micofenolato de mofetilo 0,5 g cada 12 horas.

\section{Diagnóstico}

Síndrome de superposición: Lupus Eritematoso Sistémico de inicio tardío con Síndrome de Sjögren. Probable neoplasia de gástrica. Síndrome constitucional completo. Infección por citomegalovirus.

\section{Discusión y conclusión}

La dificultad del caso que se presenta reside en diferenciar la etiología de la afectación renal. Tras confirmación de una posible causa prerrenal y posrenal, sin resolución tras hidratación y corrección de la anemia, sin poder realizar intervencionismo urológico y con presencia de proteinuria y hematuria, se decide ampliar el estudio a enfermedades autoinmunes, teniendo en cuenta el antecedente de la paciente y otras alteraciones (trombopenia y serositis).

Los resultados del panel de autoinmunidad refuerzan la sospecha diagnóstica de síndrome de solapamiento LES-SS. La realización de una biopsia renal hubiera sido útil, pero se desestimó en la paciente.

La coexistencia de SS y otra enfermedad autoinmune se presenta hasta en un $53 \%$ de los casos $^{1}$, entre ellas, la asociación con LES ${ }^{2}$. La prevalencia de SS en pacientes con LES previo varía entre un $14,8-30 \%^{3}$ y, a la inversa, hasta en un $69,2 \%$ de los casos ${ }^{4}$.

El síndrome de superposición se observa en pacientes de mayor edad, sexo femenino, con duración más prolongada de la enfermedad y menor tasa de mortalidad, respecto a los síndromes autoinmunes aislados ${ }^{3}$.
Los síntomas más frecuentes del síndrome de superposición son cutáneos y articulares ${ }^{3}$. En cuanto a la afectación renal, suele presentar un menor riesgo de glomerulonefritis respecto a los pacientes con LES aislado ${ }^{3}$ y la afectación renal en el SS suele ser poco frecuente, por lo que la presencia de fracaso renal y de pleuropericarditis en un SS puede orientar a un síndrome de superposición SS-LES².

Las alteraciones analíticas pueden también dificultar el diagnóstico por su semejanza. Puede existir positividad de anticuerpos anti-Ro en ambas patologías $^{2}$, aunque hay estudios que relacionan la presencia aislada de anticuerpo antiRo 60 kD con el LES hasta en un 30\% de pacientes ${ }^{5}$, mientras que los antiRo 52 y 60 kD se relacionarían con el SS ${ }^{6}$. La presencia de anti-dsDNA y antiSm es altamente específica para LES. Los ANA son positivos en casi todos los pacientes con LES en algún momento de su curso ${ }^{5}$.

Los niveles de complemento se correlacionan con la actividad del LES y los niveles bajos se asocian con la nefropatía lúpica, aunque se ha reportado una menor frecuencia de bajos niveles de C3 y C4 en pacientes con debut de LES tardío, si se comparan con aquellos con un debut temprano ${ }^{7}$.

Otra de las dificultades del caso fue la presencia de diarrea febril sin aislamiento microbiológico inicial, con una situación de edad avanzada y con toma de corticoides, que planteaban la posibilidad de una afectación intestinal por citomegalovirus, más frecuente en pacientes con enfermedad autoinmune subyacente ${ }^{8}$.

Finalmente, el factor limitante en el caso descrito fue la sospecha de una neoplasia subyacente, tanto por la similitud de algunos síntomas y hallazgos analíticos, así como la situación basal de la paciente, que redujo la posibilidad de realización de pruebas y terapéuticas invasivas.

\section{Bibliografía}

1. Lockshin MD, Levine $A B$, Erkan D. Patients with overlap autoimmune disease differ from those with 'pure' disease. Lupus Sci Med. 2015; 2(1): e000084. doi: 10.1136/lupus-2015-000084.

2. Zufferey P, Meyer OC, Bourgeois P, Vayssairat M, Kahn MF. Primary systemic Sjögren syndrome (SS) preceding systemic lupus erythematosus: a retrospective study of 4 cases in a cohort of 55 SS patients. Lupus. 1995; 4(1): 23-7. doi: 10.1177/096120339500400106.

3. laccarino L, Gatto M, Bettio S, Caso F, Rampudda M, Zen M, et al. Overlap connective tissue disease syndromes. Autoimmun Rev. 2013; 12(3): 363-73. doi: 10.1016/j.autrev.2012.06.004.

4. Manoussakis MN, Georgopoulou C, Zintzaras E, Spyropoulou M, Stavropoulou A, Skopouli FN, et al. Sjögren's syndrome associated with systemic lupus erythematosus: clinical and laboratory profiles and comparison with primary Sjögren's syndrome. Arthritis Rheum. 2004; 50(3): 882-91. doi: 10.1002/art.20093.

5. Bertsias G, Cervera R, Boumpas DT. Systemic lupus erythematosus: pathogenesis and clinical features. In: Bijlsma J (ed). EULAR textbook on rheumatic diseases, London: BMJ Group, 2012,476-505.

6. García-González M, Rodríguez-Lozano B, Bustabad S, Ferraz-Amaro I. Undifferentiated connective tissue disease: predictors of evolution into definite disease. Clin Exp Rheumatol. 2017 Sep-Oct;35(5):739-745.

7. Alonso MD, Martinez-Vazquez F, de Teran TD, Miranda-Filloy JA, Dierssen T, Blanco R, et al. Late-onset systemic lupus erythematosus in Northwestern Spain: differences with early-onset systemic lupus erythematosus and literature review. Lupus. 2012; 21(10): 1135-1148. doi: 10.1177/0961203312450087.

8. Ko JH, Peck KR, Lee WJ, Lee JY, Cho SY, Ha YE, et al. Clinical presentation and risk factors for cytomegalovirus colitis in immunocompetent adult patients. Clin Infect Dis. 2015; 60(6): e20-26. doi: 10.1093/cid/ciu969. 